\title{
Modelling the Clinical Risk: RFID vs Barcode
}

\author{
1Vincenzo Di Lecce*, ${ }^{1}$ Marco Calabrese, ${ }^{2}$ Alessandro Quarto, ${ }^{3}$ Rita Dario \\ ${ }^{1}$ Politecnico di Bari - DIASS \\ ${ }^{2}$ myHermes S.r.l \\ ${ }^{3}$ Hospital Unit "San Paolo" ASL/Ba. \\ ITALY
}

\section{Introduction}

This chapter proposes an approach that can improve the identification of patients, products, equipment and so on in a hospital. The aim is to better the management of the clinical risk by automating the process through the use of Radio Frequency IDentification (RFID).

The clinical risk management refers to the procedures for avoiding risks associated with direct patient care. It is the analysis about the probability of a patient being victim of an adverse event or inconvenience resulted, although involuntary, from improper medical care provided during the hospitalization. Research defines that the common denominator of almost all adverse clinical events is the lack of the patient traceability. In the specific context of a hospital, "traceability of patients" means managing a whole set of input and output data related to those processes defined as critical for clinical facilities.

In this work we will discuss a model to archive and manipulate data concerning the proposed system. RFID is, in fact, identified as a method for storing and retrieving data remotely by using small and cheap devices called RFID tags or transponders. For applying this technology in hospital environment it is necessary to build a network of these objects, each one with a unique number. So, only if there is a valid model to manage the produced output, one can easily retrieve information and realize an interesting use case.

In the proposed model, the adoption of an active instrument for automatic patient identification and assistance can be considered as the central entity of the process: "medical treatment of a patient", this includes potentially every activity from initial consultation through discharge of a surgical patient. In particular, this approach allows for simplifying the reality in terms of observable entities and supporting a higher degree of interconnection among those sections that traditionally are called "functional areas" of the hospital organization. Indeed, a lot of different actors characterize a clinical environment and the use of RFID seems to be capable to track all of them and optimize their interaction (Liao et al., 2006). The proposed RFID applications are targeted to the:

- Verification of positive patient identification. This is realized by means of a smart wristband for each patient. The tag contains information about the patient name, date of

${ }^{*}$ corresponding author 
birth, surgical information, allergic reactions, medication requirement, blood type, health condition;

- Monitoring of surgical equipment before and after the operations. This application is aimed to grant a more efficient knowledge of the hospital instrument thus avoiding the stealing and misuse of equipment;

- Asset identification, such as blood transfusion, pharmaceutical units, charts and specimen;

- Bed inventory;

- Tracking of hospital staff and patients. Scientific works demonstrate, in fact, that geographical location of transponders is realizable by using a network of RFID readers in an indoor area. The idea is to divide the area in a set of sub-regions and read the power level each tag produces in relation to a particular reading station (Sangwan et al., 2005).

This paper also deals with the impact that the application of the RFID model produces on the traditional organization of a hospital unit. To this end, it is worth noting that the staff work is partially influenced by the new process organization. Therefore, it is clear that for realizing a cost/benefit analysis it is necessary to consider the importance of training and transition.

The outline of the chapter is as it follows. Paragraph 2 introduces the definition of clinical risk management, sketches an abstract model of it and provides an overview of ICT impact on the healthcare process with particular reference to barcode and RFID technologies as they have been used and implemented in the healthcare according to recent literature. Paragraph 3 makes a comparison between barcode- and RFID-based data models in order to enlighten the different perspective and benefits introduced by RFID technology in the patient care. In particular, the shift from a model based upon the concept of medical record to a new representation where the patient is at the center of the healthcare process is thoroughly examined. This change allows for a step-by-step reengineering of the healthcare process from within, which is extremely difficult using the traditional approach. In addition to traceability requirements in fact, an RFID tag (positioned, for example, in the patient's wristband) provides a real-time event trigger mechanism, which is very useful when a complete overview on the instantaneous state of the healthcare process is needed. A real-world example of how RFID can be easily integrated in the existing hospital environment is then presented in Paragraph 4. Paragraph 5 draws conclusion.

\section{The Clinical Risk Management}

The public healthcare recently and before the private one has been giving particular attention to the question of the clinical risk management. With reference to the Italian situation, for example, the prescriptive requirement for this issue may be due to the need to confirm the healthcare process for delivering quality services and therefore the recognition of those health protocols being valid for the institutional accreditation of all hospitals. The same consideration may be extended to most of Western countries as well. At the core of the quality process within the healthcare system there is also the traceability of any healthcare action referred to patients/users; because just the lack of such requirement is one of the main causes of the "clinical error", hence it is often responsible of high clinical risk. 
The "traceability" is defined by the norm UNI EN ISO 9000:2000 as the ability to trace the history, use or location of what is under consideration. In a hospital the maintenance of traceability is a critical concern involving transversely those processes controlled by the structure organization system, whether it is based on traditional paper records, both in the case of a fully computerized management system, as well as in all hybrid-type situations. In any case, the traceability maintenance is based on an appropriate set of information relating to input and output data of certain processes identified as critical.

Some of the most critical processes within a healthcare structure concern the management of a large amount of information about drugs to be administered to patients, the examinations to be performed, the results of the tests, the diagnosis and clinical picture of patients until their discharge from a hospital department. The criticality index of processes depends on the criticality of the patients and their ability to communicate in a non-linear but actually exponential way.

The most advanced technological method, which is currently in use and able to minimize the risk by an error in the traceability process, without necessarily revolutionizing the entire management system of the healthcare setting organization, is the one involving the use of bar-coding printed on labels (barcode), which in turn are applied on the patient's medical records or other (infusion bags, medication, wrist bracelet). The coding contains a minimum set of information that can be read by optical devices connected to an appropriate computer system.

A suitable alternative is provided by RFID technology, recently introduced in clinical engineering, which seems to offer considerable advantages in terms of reliability, efficiency, versatility and ability to provide information.

In comparison with the old barcodes through this technology a patient that wears a bracelet containing a RFID transponder, is physically and uniquely included in the management process of clinical data, while with traditional systems the patient can be recognized only by using personal data.

\subsection{Modelling the Clinical Risk Management}

The debate about the clinical risk began in the 70s in U.S. in order to prevent the increasing trend in compensation claims by patients who had suffered damage as a result of errors in delivering the treatment they were subjected to. The most significant system, which is mentioned as an example of the national healthcare monitoring is the Australian Incident Monitoring System (AIMS), with 50,000 reports in 2001, 26,000 of them were sent directly from health facilities, while the remaining part by the operators, often anonymously, to the departments in charge. In Italy, specifically in Emilia Romagna, a similar system, which involved 39 units from 5 local health authorities of the region, was tested in 2001.

The risk management is about the identification of risks associated with the organization activities and the use of appropriate and adequate practices to prevent these risks or minimize their effects. The fundamental elements of risk management are: risk identification, risk analysis, risk treatment and monitoring. In this context the main modalities of risk identification are: the use of administrative and information data, the incident reporting and structured review of health records. 


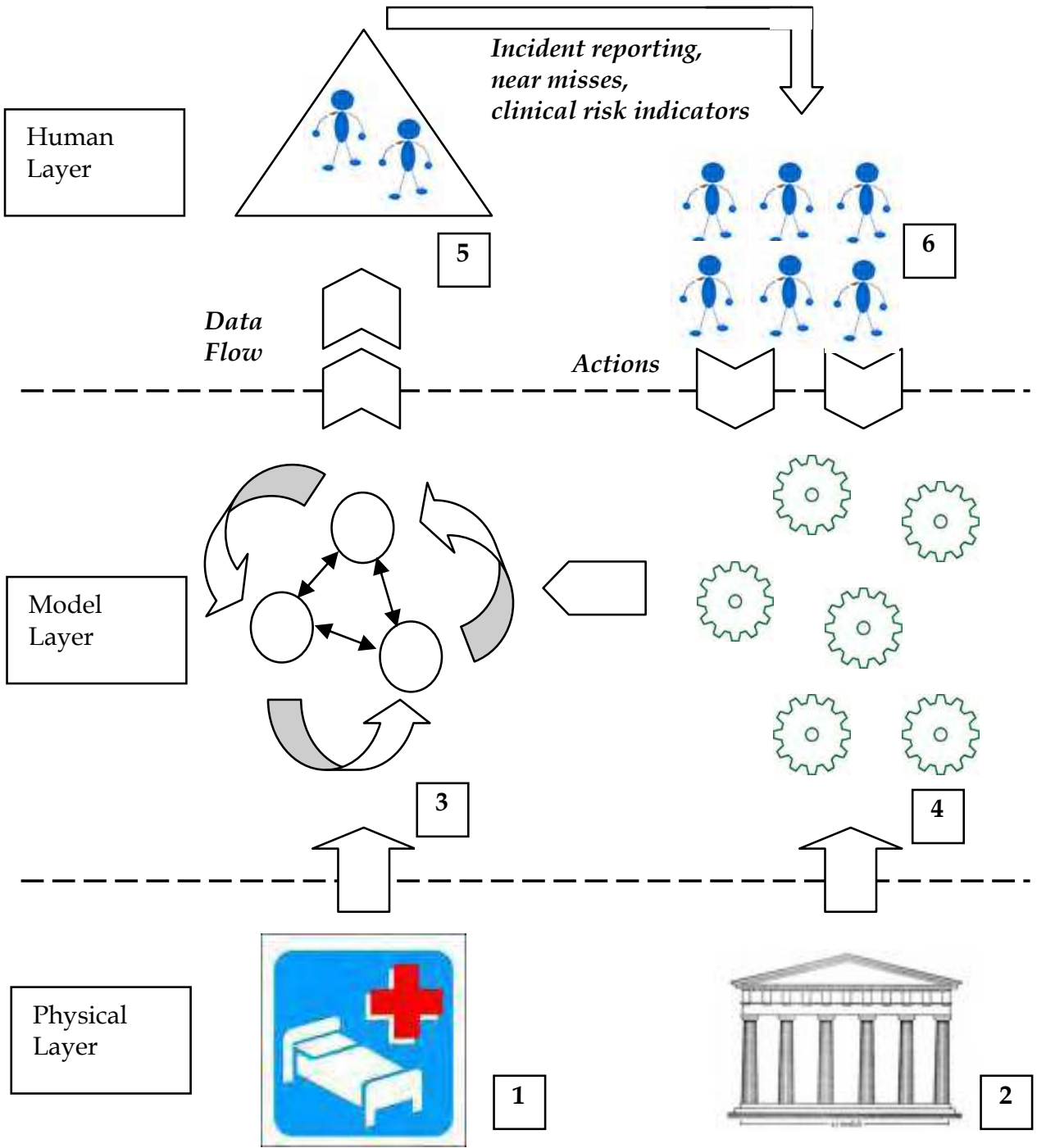

Legend

1. Healthcare Centres: hospitals, clinics

2. Normative Institutions: ministry of Health, Academies

3. Processes

4. Norms: protocols, guidelines

5. Supervisors

6. Stakeholders: medical staffs, patients

Fig. 1. Clinical Risk Management layered representation. 
These data are useful to perform an assessment of adverse events that have already occurred (misses) or that are potentially verifiable (near-miss).

In order to produce a complete model of the risk management, a preliminary investigation on which variables are needed and what semantics they have is due. In-field data actually measured in real-world scenarios need in fact to be conceptualized within the ontological framework of the problem. As a rule of thumb, the more complex the underlying ontology in terms of numbers of independent concepts, the more the number of independent variables that should be considered. In computer science, ontology is defined as "a specification of a representational vocabulary for a shared domain of discourse - definitions of classes, relations, functions, and other objects" (Gruber, 1993). In other words, ontology can be considered as the formal specification of conceptualizations of certain domain knowledge. Ontology models the world of interest through assertions in a given language. Well formed assertions require a grammar, i.e. a way to assess the correct order of sequences of symbols used to describe the problem under consideration by means of formal rules. This would require an accurate ontological approach which goes beyond the scope of this work and will be investigated in future work on the subject.

For the scope of this chapter it is sufficient to describe the clinical risk management using a three-layer representation as depicted in Figure 1. The base layer accounts for places and institutions that are involved in the healthcare process. Hospitals and clinics are the physical places where processes come in action, while the Ministry of Health and other accredited institutions are responsible for providing formal informal guidelines and protocols that have to be implemented by medical operators. Processes and norms characterize the Model Layer: if correctly managed, they guarantee a suitable match between clinical theory and practice. At the upper layer two main groups can be found: stakeholders (such as medical staff and patients) and supervisors (that can be internal or external to the supervised structure depending on national healthcare systems). This last group supervises correct clinical risk management through in-field data collecting. In Italy, for example, this institution is called "Direzione Sanitaria" (Health Care Directorate) and works as a local control body in cooperation with the Ministry of Health. Supervising bodies are generally involved in the evaluation of the clinical risk through appropriate indexes which are produced through the accurate analysis and interpretation of recorded historical data. The acquisition of these data is generally achieved through incident reporting provided by medical staff.

\subsection{Computerization of hospital healthcare data for clinical risk management}

The automation of health records helps reduce the risk of error in healthcare, but contemporarily it involves new elements that must be analyzed; this implies necessarily and primarily a management of organizational flows referred to the clinical process. The automation procedure includes various components related to two areas and in each of them it is therefore possible to highlight the main sources of clinical errors:

A) Information Generation

a. The information management operators

b. The evidence of errors or omissions

B) Information Use

a. The interpretation of the data

b. The integration of information bases 
c. The availability of the data

d. The redundancy of information

e. The storage of information

From this it appears that all the data being specific to an information system interact either directly or indirectly in the safety of clinical processes.

Information Generation. The first effect of computerization is the change in the way of recording information. In an ideal path it is necessary to provide for the complete elimination of paper-based materials and at the same time it is important that who performs the healthcare action, should be also in charge of registration, for example: the doctor who prescribes a treatment, will have also to carry out the same operation by means of an information system directly. Therefore, it is clear that it is not a true automation of the information flow where there is still a stage of the clinical process based on paper records, such as a prescription made first on paper and then subsequently computerized.

From the perspective of risk assessment, the computerization of data at a later phase leads to the introduction of such errors of interpretation or transcription, which remain hidden for the following user of the system, since these risks cannot be manageable and much less controllable.

Information Use. Automation facilitates the access to the data, which are presented in a modality that is more different than that of the paper: if ergonomic guidelines and architectural aspects for the user interface design are not taken into account, there will be the risk of an incorrect interpretation of the submitted data thus generating an error. The integrity of the data and its continuity (backup, redundancy and disaster recovery) will be protected by the adoption of universally recognized standards in the analysis process.

In 2005 the process of incident reporting was activated in all public health facilities referring to the Local Health Authority 4 in Chiavari (Genoa, Italy) introducing a risk warning report. 660 records between the years 2005-2007 have been analyzed, 10\% of these are about errors or near miss related to the identification of patients. The highlighted issues were:

1. Non-application of the patient's identification bracelet for accessing to the operating room;

2. Misidentification of the patient for the therapy;

3. Misidentification of the documentation: reports, examinations, labels, etc..;

4. Misidentification of patient to perform non-invasive care practices

Furthermore, from this clinical experience, it has been shown that the critical processes are represented by the admission of patients in the structures of the Local Health Authority, the access of the patient in the operating room and the therapy administration.

In 2007, in Apulia Region, the Regional Health Agency has launched a survey about procedures and technologies for the patient identification in the main hospitals. In particular, the required data referred to identification procedures such as:

- identification of the patient;

- Identification of diagnostic reports;

- Identification of biological samples;

- Identification of drugs and therapies;

- Identification of operators. 
From a technical point of view, the Apulia Region refers to three classes of encoding devices that should be present in the hospital:

1) Clear Information: The identification modality is based on the presence of some essential information (second name, first name, date of birth, tax code, etc..) readable in text mode directly by the operator and usually included in a wristband applied to the patient;

2) Barcode: identification system using a bar code system;

3) RFID: this latter alternative shows significant advantages in terms of reliability, efficiency, versatility and ability to hold information, as compared to the old bar code devices. In fact, with this technology, the patient wearing the wristband containing an RFID device is physically and unequivocally included in the computerization process of clinical data management.

The employment of such objective identification systems is of priority importance especially in those wards considered as the most critical ones for the clinical risk due to the sensitivity of patients as well as the delicacy of assistance procedures such as those of the intensive care, neonatal unit and transplant patients (Di Lecce et. al, 2008).

The results of this investigation are still in the experimental phase of acquisition and processing for the next operational provisions and the relevant evaluations will be acquired by the Working Group under the Regional Plan for the Electronic Healthcare activated in 2006 in the field of ICT (Dario et al., 2007.a).

Rothschild and colleagues (Rothschild et al, 2005) have studied in a medicine department of a hospital in Boston as the most sensitive patients hospitalized in intensive care units are more susceptible to the consequences derived from the effects of adverse events. In the same hospital, (Lehmann et al, 2005) have studied iatrogenic events, occurred during the administration of drug therapy, as the main source of clinical error.

An essential example of risk management analysis, from which the need of applying the RFID technology in a hospital arises, is represented by the database design for the hospital S. Giovanni Battista in Turin (Rapellino, 2005). This project has started since 2000 and contains data related to the identification of unfavourable events occurred within the hospital. From that work presented in 2006 it results that $47 \%$ of adverse events is due to technical-structural and organizational problems of the healthcare structure.

One of the most risky procedures for the possibility of falling into the therapeutic error refers to the administration of blood products. The barcode recognition on blood bags is one of the oldest and most useful traceability system that is worldwide recognized. Even Turner CL. et al. (Turner et al., 2003) had already shown in 2003 as the implementation for the identification of the patient, subject to blood transfusion, by using barcode system rather than the verbal or written modality has led to a significant increase in safety of blood transfusions.

In 2006 Wicks and colleagues (Wicks et al., 2006) described the potential benefits, application areas, the implementation changes of health care processes and the corresponding structural strategies in the use of RFID technology in a U.S. hospital department.

Some Dutch authors (Friesner et al., 2005) have recently presented again the need of a careful analysis of audit by users, before and after the introduction of each new technological application in hospital. In 2004, an American unit of intensive pneumology has conducted a survey of incident reporting; Osmon S. and collaborators (Osmon et al., 2004) have stressed that most of medical omission and commission of adverse events 
occurred outside the department of intensive therapy, especially during the emergency transport of patients among the different floors of the Hospital.

An application model made with the international partnership between hospital units in Palermo-Sicily Region and the University of Pittsburgh Medical Centre, represents the experience described by the authors in 2007 (Brenni et al., 2007), i.e. an information system also extended to electronic medical records. The system presents the integration of RFID technology by means of disposable bracelets with single sign-on rewritable passive-type tags applied at the access time for the authentication of patients.

\subsection{Related Works about use of RFID and Barcode in Clinical Risk Management}

The hospital belongs, by its nature, to the framework of complex structures. The typical activities of this context regard concepts such as: traceability, reliability, security. Historically the use of protocols (therapeutic, surgical, pharmaceutical, logistics etc.) has characterized such activities. The matter regarding hospital logistics as operating framework, (i.e. knowledge acquisition of changing processes that take place within the hospital for the reorganization and reengineering of structures), or as quality improvement (i.e. safety and appropriateness of clinical care services) has been under consideration for many years. Basis of this operational framework is typically the traceability, which is classifiable in hospitals as:

- Traceability system of wards;

- Traceability system of emergency wards;

- Traceability system of pharmaceuticals (From the delivery of the drug produced by the manufacturer to the production of the single dose);

- Traceability system of medical devices;

- Traceability system of blood bags;

- Traceability system of implantable devices.

Numerous experiments carried out in the early nineties have validated this approach in view of technological supports in use. Current tendency is to focus, in particular, on the management of physical paths of patients (patient flow). A more rational management of the physical patient flow could solve the typical hospital problems such as: delays, long waiting times, queues, erased interventions, patients placed in inappropriate care setting, nursing staff under stress, waste and high costs etc. In this regard, a remarkable experience is that referring to the surgical patient in Boston Medical Center.

In Italy the USL 8 (Local Health Unit) of Asolo Hospital and Cardinal Massaia Hospital belonging to ASL 19 (Local Health Authority) in Asti, (both of them date back to 2006 ) may also be considered as qualifying experiences. Especially that of Cardinal Massaia Hospital (Asti - Piemonte Region, Italy), where the experimentation, started in 2006, has involved the wards of Pediatrics and Cardiology; here RFID wristbands, encoded during the First Aid in Emergency ward, are assigned only if the patient must be hospitalized in the two enabled wards. While in other wards barcode technology is still used. This experience also allowed for a comparative evaluation of the two technologies.

Bates et colleagues (Bates et al., 2001) analyze how the use of information technology can be a practical support for the reduction of accidents involving hospital patients. Among the criteria for the reduction of clinical risk, there is the need to develop efficient communication information systems able to involve hospital operators. The main difficulty is, in fact, 
interpreting and rapidly exchanging data produced by each different health care settings, such as analytical laboratories or pharmacies. This separation of information is considered as the biggest barrier for achieving a real clinical risk reduction. It is therefore important to underline the concept of creating a channel for asynchronous data exchange, so that information flows, which are extremely important and characterized by a strong urgency,( i.e. critical results derived from a particular analysis cycle) can be exchanged with a minimized latency time. It is similarly important to underline the use of technology for identification of patients, drugs and hospital instruments, by means of barcode system applications, in order to significantly reduce accidents caused by poor communication.

The comparison between barcode and RFID technologies in patient identification is realized in (Aguilar et al., 2006). They underline the fundamental differences between them: barcode are scanned one at time and RFID can be scanned continuously by one reader; a barcode can be printed and cannot be modified whereas an RFID tag can be re-writable; barcode requires line-of-sight and RFID not, so RFID can be used in applications that aim to eliminate human intervention; RFID presents more complicated forms of data protections and encryption than barcode; barcode devices are typically cheaper than the RFID ones.

Young (Young, 2006) discusses the possibility of optimizing medical assistance activities by using RFID and barcode technology. In particular he has suggested a coordinated use of the two technologies in order to achieve an appropriate balance between the implementation costs and benefits derived from them. The use of RFID technology, in relation to a high unit cost and considering the classification of patients and irregular material is really advantageous thanks to their reusability and ease of reading data. The use of barcode system can be seen as the solution for producing regular material inventories, taking into account the low cost but, at the same time, the greater difficulty in querying these systems due to their passive nature.

Perrin and Simpson (Perrin \& Simpson, 2004) highlighted how healthcare organization should make special efforts to adopt the so called Auto-ID/Bar-Code Enable Medication Administration system, either it employs active technologies or it employs the passive ones. The major difficulties concern the need of re-engineering organization procedures. On the other hand, by drastically reducing adverse events in the hospital there can be several benefits (also economic), the reduction of legal costs for services and the great satisfaction experienced by personnel in getting the reduction of accidents, as well as the ability to optimize the work organization of each functional sub-area thus increasing in efficiency.

Jiang and colleagues (Jiang et al., 2005) analyze the use of barcode and RFID technologies in management of blood. In particular, the research would propose an information management system to reduce the AIDS diffusion caused by the transfusion of infected blood. The authors start investigating the proprieties of a traditional information system based on barcode. The main disadvantage is that the barcode on the blood bag carries less information and cannot be reused, so it is not possible to store dynamic information about donors. The proposed solution to the problem is based on the use of RFID architecture. Jiang proposes the use of a donor's smart card to store all the details about the clinical life of donors. The research analyzes also the management of blood bag. The traditional barcode system is characterized by a static information management; so when a blood bag is sent to a hospital and the blood is used then its label is usually discarded. So the blood center cannot know what has happened to the blood. With the use of RFID, doctors can avoid 
some problems of quality about the blood during transfusion and all data can be written on the smart chip.

In (Fuhrer \& Guinard , 2006), authors present how RFID technologies can contribute to build a smart hospital by optimizing business processes, reducing errors and improving patient safety. In particular, the paper underlines the importance to adopt an information system to manage usefully the data that the RFID technology can produce. In the proposed smart hospital many assets and actors would be tagged by RFID: the medical equipment and all the devices should contain an embedded tag; all the doctors, nurses, caregivers and other staff members should use a personal smart badge; each patient receives a wristband with an embedded RFID; all the paper medical files and other documents are tagged with selfadhesive RFID labels; the blister packs, other drugs' packages and bags of blood contain RFID labels. To produce dynamic information about the hospital activities RFID readers are placed at the entrances and exits of the smart hospital. Additional RFID readers are in each operating theater and in most important offices and strategic passages. At the same time a set of RFID palm readers is distributed to the staff members. Using this architecture, authors propose the possibility of producing XML real-time in correspondence to hospital events and store all the information in the information system.

Also Wu B. et colleagues in $(\mathrm{Wu}, 2005)$ analyze the application of RFID sensor networks in a smart hospital to improve efficiencies in operational aspects. The use of the RFID sensor nodes can generate large amounts of data. All the data are real and produced real-time, but they are not understandable without an appropriate middleware to manage them. The authors propose a middleware to connect the sensor networks to the real hospital applications.

In (Al Nahas \& Deogun, 2007) a survey on the use of technologies for the patient identification is conducted. The main objective for the adoption of these technologies is the reduction of the clinical risk. Authors analyze barcode solutions, largely used prior of the RFID. It is estimated that the 70 percent of all medication containers had barcodes in 2006 and also staff and patients can be identified by barcodes. Limits of barcode refer to the fact that they are sensible to wrinkling, tearing and wetting and that cannot be used for real-time tracking. So the RFID looks like the solution for clinical tracking application. RFID, in fact, does not require line-of-sight for scanning and are resistant to tearing and moisture. Another advantage of RFID is that active tags can be used for tracking equipment, staff and patients in real-time.

The contemporary use of barcodes and RFIDs is proposed in (Sun et al., 2008). In this case, the problem under analysis concerns the reduction of medication error. The authors move from the consideration that the RFID is a good solution for patient identification in hospitals; however due to the high cost of tags and readers, most of healthcare industries hesitate to introduce them. So the proposed system requires that each unit-dose medication has a barcode and each patient needs to carry a wristband embedding a RFID tag. So the hospital information system works by managing the information produced by both the technologies and read by staff PC and PDA.

In (Clarke \& Park, 2006) authors analyze the impact that the adoption of new technology has on the work of a hospital facility. The authors underline that the physician and staff satisfaction is heavily influenced by the degree to which the new work process disrupts existing practices. So the hospital management would prefer changes that can improve the workflow and information within existing practices. If it is necessary to adopt a disruptive 
change, also a cost planning and training must be considered. Typically the IT-based process changes involve the introduction of new technologies that can be difficult to integrate into the traditional workplace and workflow. In order to avoid these well known problems, the authors propose the use of a low invasive and semi-automatic technology such as the RFID. This solution can be used for passive tracking of people, objects and documents of a clinical environment.

$\mathrm{Ni}$ et colleagues produce a study and a survey about a number of wireless technologies that have been used for indoor location sensing. In particular they focus on the use of RFID technology and propose a prototype called LANDMARC to realize an accurate indoor location sensing system (Ni et al., 2004).

\section{Healthcare - Data Model}

Hereinafter ER models will be presented to describe the health procedures for patient identification and assistance during the hospital stay. In particular, two representative models have been identified: one for encoding barcode type and the other based on RFID systems.

The main difference between the two technologies refers to the operating principle; it will be passive in the case of optical systems and active for Radio Frequency Systems; this difference impacts also on the hospital organization in relation to the quality of health services for the patient.

The transit from the Barcode model to the RFID model proposes a strong analogy with the evolution of the World Wide Web. The biggest innovation is, in fact, that an active technology transforms the patient from a simple information consumer to a data producer.

\subsection{The traditional Data Model}

The Feature of barcode model is that it can identify a multiple number of organizational subsystems and each of them refers to a different functional area for the patient assistance.

In particular there are specific sub-areas describing:

- Medical services: the main entity is the "medical staff" actively involved in the production of tuples describing other entities: The performed "physical examinations", the issued "prognosis" and the associated "therapy". Of course, all information are stored in "medical record" in conformity with the time of production;

- Pharmacy services, laboratory analysis, diagnostic services: it is the sub-organization describing activity that the pharmacy staff performs in relation to the interpretation of medical record and medicine box packaging for the patient therapy. In this information system there is a second active entity, established by the "paramedical staff" responsible for the distribution of medicine boxes;

- Technical operating services: it is the model of functional reality associated with "hospital activities" conducted on the patient, such as: interventions, X-rays, ECG and other forms of health investigations. The active entity of this system is identified in the "operators" responsible for the different " hospital activities " associated with the production of specific outputs, that, as the diagrams show, are called: "report";

- Management control. 


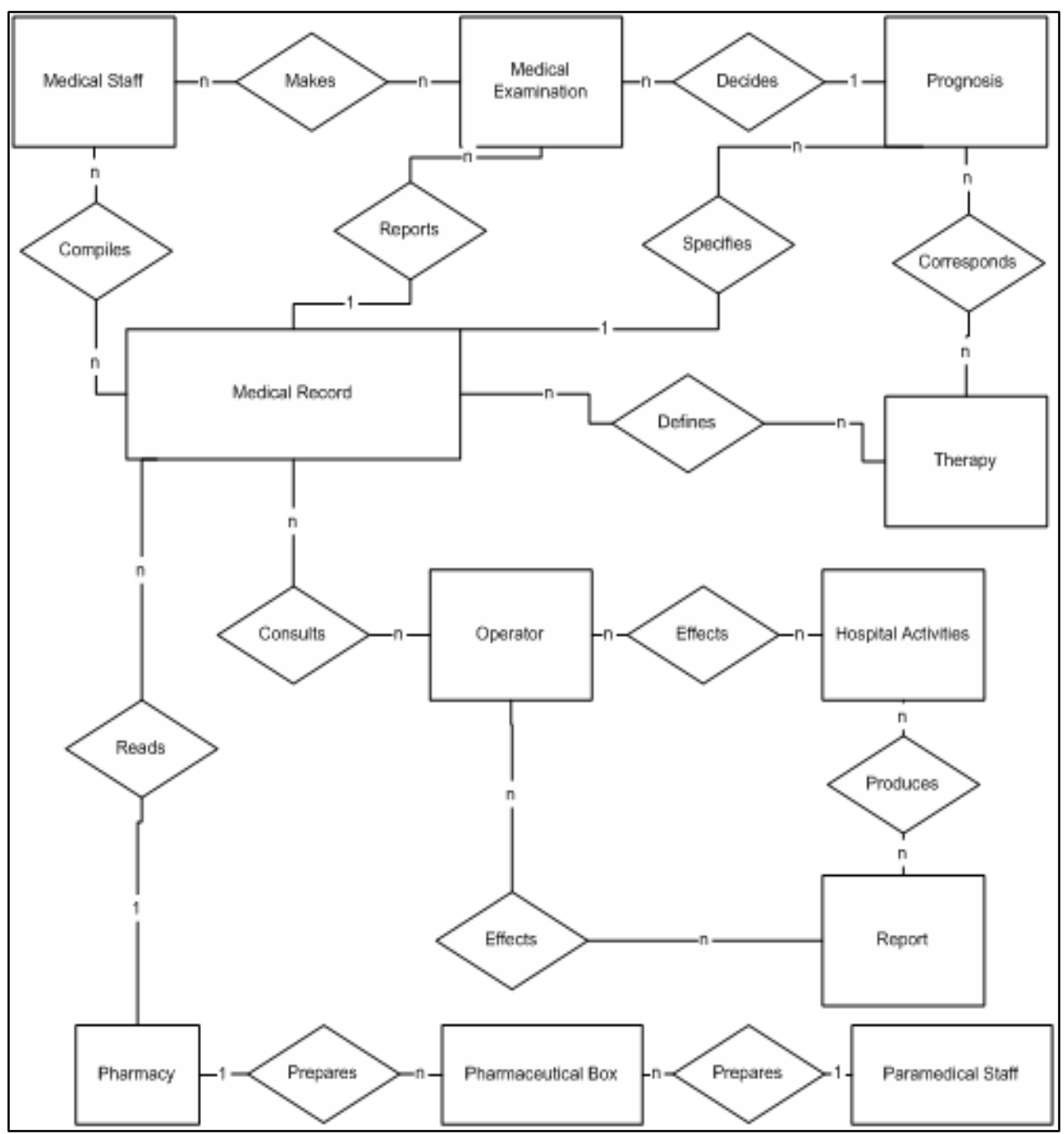

Fig. 2. - Medical system using Barcode autoidentification technologies.

The aggregation of summarized outputs from the produced information flows can be synthesized in the "medical record", i.e. a single centralizing entity allowing for the storage, in a strictly sequential mode, of data produced by each functional area in relation to the same patient (Figure 2). In other words, the traditional notion of "medical record" can be interpreted as the only entity in common among many information systems. Such a representation can be easily enhanced to support those passive technologies for the authentication of the patient, who is not characterized by any explicit diagrammatic presentation, but is an attribute of the before mentioned instrument for connection. 


\subsection{The "active patient" Data Model}

The adoption of RFID as a tool for automatic patient identification can be considered as a central "active" entity of the whole model (Roark \& Miguel, 2006). The alternative, presented in the associated diagram (Figure 3), is characterized by a simplification of reality in terms of observable entities; this means reducing the risk of information duplication that the traditional model shows (Brenni et al., 2007).

On the other hand, it is important to note a higher degree of interconnection among those that have been previously called "functional areas". In this model of reality, in fact, it is no longer possible to identify a clear separation among them, but their integration is recognizable and it is mainly identified in the "patient" and "health condition". The diagram is basically characterized by the disappearance of the "medical record" concept , seen as an instrument for getting information about activities produced by the system in relation to the patient, who it is associated to, and therefore the new entity "health condition" replaces the medical record and refers to the patient condition in a specific moment. Since the "health condition" is linked by a univocal relationship to the "patient" that it describes, it is clear that there is a direct relationship with the activities related to: "physical examinations" made by the medical staff; drug management (prescription, administration) made by the pharmaceutical staff and "performance" of other hospital operations for monitoring patient health condition (analysis and investigations) together with other correlated actions.

The comparison between the two proposed alternatives highlights the characteristics of simplification introduced by the RFID model, without having any increase in approximations made in view of the described reality. On the other hand, the presence of separate functional areas that are typical of barcode system draws attention to the problem of risks associated with information duplication. Indeed, the univocity of the chosen communication channel ("medical record") determines that the contact among different information flows can occur only after or before the processes have been developed by each functional unit (Dario et al., 2007.b).

So in relation to the clinical risk, asynchronism of communication can lead to lack of communication phenomena, which can be associated to the delta time characterizing the accesses for reading or writing the "medical record". However, the presence of a single communication node determines a possible break thus leading inevitably to the collapse of the whole macro system with all the relative risks connected to this (Brenni et al., 2007). In an interconnected model, similar to that using RFID technology, it is ensured that the temporary absence of information flow does not inhibit data exchange to all other entities of the system, which can continue to operate even under reduced conditions. The application of RFID model can be evaluated as optimizing also in terms of operation traceability. Certainly, a system producing a single intra-hospital communication output and characterized by sequential writing access, can easily suffer from the possibility of being improperly altered, differently from what actually occurs if the exchange of data and information among the operators takes place in real-time (Bates et al., 2001).

In conclusion, using such technology as RFID would mean several advantages to health care processes with only a slight reengineering of specific structures. A more rational use of hospital information systems could allow for a better organization of clinical data. Moreover it is noteworthy to remember the launch of RFID and Wi-Fi compliant transponders on the market. These would allow for unifying access points, thus simplifying them, (from a 


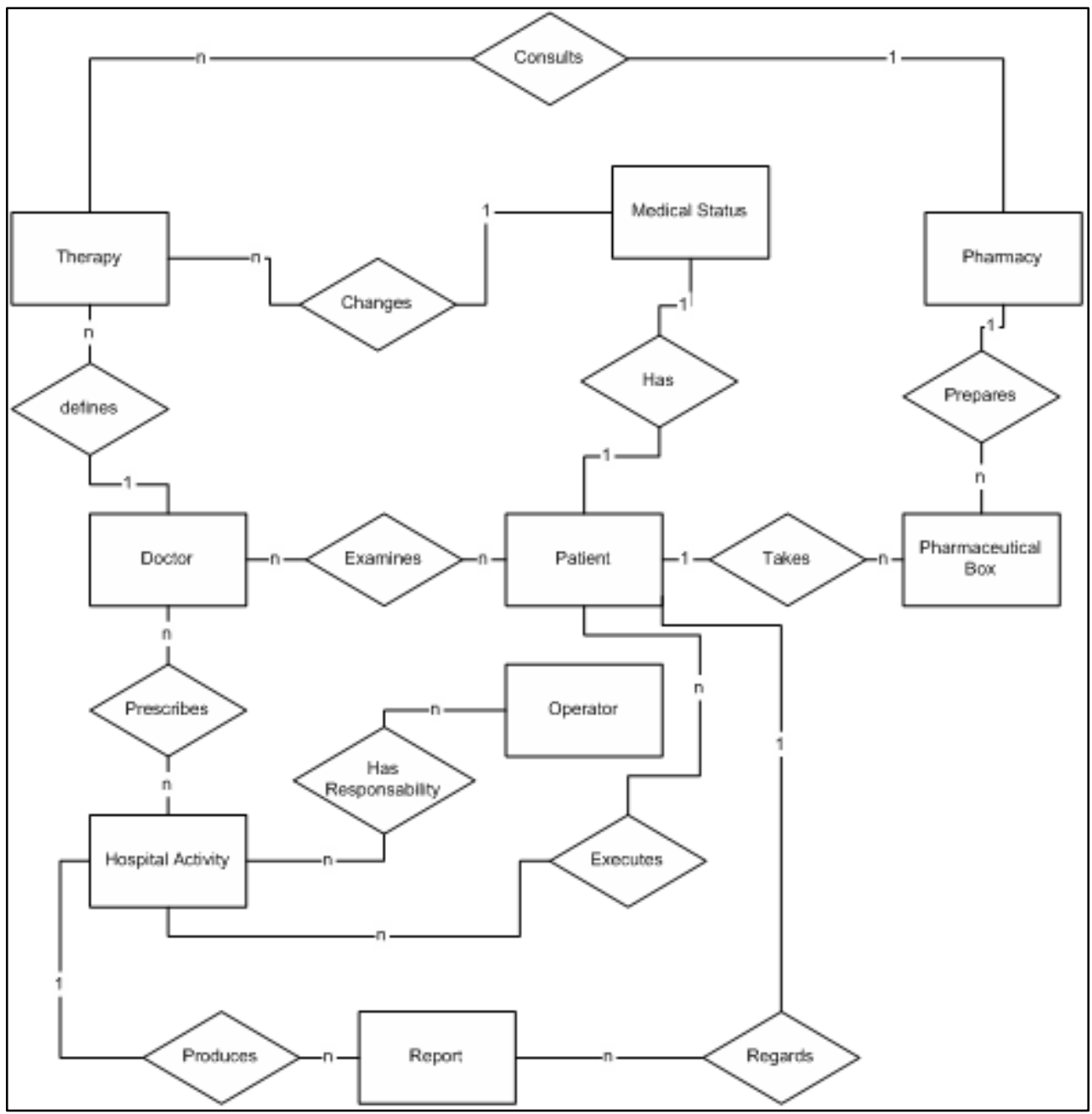

Fig. 3. - Medical system using RFID autoidentification technologies.

specialized portal to a pervasive wiring) and obtaining, through triangulation techniques and / or the power density calculation, the precise location of monitored entities. It is well known that the process for examining the clinical risk must start from identification of risks analytically, and therefore define the potential risks according to predetermined international grading scale in order to organize and implement the risk reduction project (Perilli, 2007). A possible solution for reducing the incidence of errors related to "mistaken identity" seems to be the use of identification wristbands with advanced RFID or barcode systems at the time of admission to hospital.

Of course this solution cannot be considered as the only or essential strategic element in the management clinical risk for the patient safety. To sum up, the use of an identification advanced system can not exclude the introduction of "best practices" relating to the 
implementation of the system for "incident reporting" and realization of campaigns focussing on specific issues, which are crucial to hospital security for both operators and citizens.

\section{Advantages in using RFID-based healthcare systems: real-world example}

The need to control and rationalize health expenditure for hospital services in Italy has led to the systematic recording of health data, through the introduction of the so-called S.D.O. "Scheda di Dismissione Ospedaliera" (Hospital Discharge Card), and the new reimbursement system by means of pre-determined charges as well as health services provided through D.R.G. (Diagnosis Related Group). The inclusion of the S.D.O. in the national health system, both public and private sectors, is regulated by the Ministerial Decree dated December, 28th 1991, and it has really replaced the previous system of data gathering, based on ISTAT (i.e. the Italian National Institute of Statistics) forms since 1995, also because of the dual role that currently this new document has: clinical summary and propaedeutic tool for billing the servicesperformed Although the D.R.G system classification can be traced back to 1983 in the U.S., its inclusion in Italy became effective only with the Ministerial Decree dated July 26th 1993 (the "Regulations of information flows about people discharged from public and private hospital institutions"). The introduction of S.D.O. and D.R.G. system brought about significant and radical changes in the organization of both public and private health facilities. The initial resistance to this change, due to the lack of competence in filling in these cards, with the different operating model, was overcome by launching a "navigation view" (as it happens for dialysis health services in Apulia Region), and this means that it is necessary to fix, update, manage more times the filling in procedure of the Hospital discharge card and the application of D.R.G. The completeness, accuracy, and timeliness of transmission from regions to the Ministry of Health in filling in the S.D.O. are obviously needed to monitor the regional and national progress of public and private health activity and therefore the following hospital expense.

\subsection{Beyond "navigation view"}

The technique of navigation view includes the non-systematic solution of medical errors (corrections, verification of the previous cases etc.) that are not always effective and, at the same time can be highly risky due to the "propagation of errors". The goal is the "automatic navigation" by using a series of information systems that can support the medical activity while filling in the S.D.O. and when assigning the D.R.G. This effort should be aimed at obtaining, real-time, the projection being as much accurate as possible in relation to the clinical diagnostic protocol that the patient follows during the period of hospitalization. The current legislation provides for the activation, through the health management administration, of systems for checking the completeness, timeliness and quality of the information contained in the S.D.O. The guidelines of the Health Ministry in June 1992 gave great opportunity for controlling the internal organization of the data flow system. However, up to the authors' knowledge, a comprehensive engineering approach to healthcare workflow management in Italy is quite far from being achieved. The proposed RFID-based approach moves towards reducing this gap.

S.D.O is uniquely attributed to the clinical history of one single patient's hospitalization. This means that its underpinning data model is in accordance with the ER diagram depicted 
in Figure 3 which adopts a patient-based view. A typical S.D.O. in Italy consists of two pages as shown in Figure 4 and 5. The first page is referred to patient's identification data and summary information about his/her hospitalization, while the second page reports on events related to diagnostic and therapeutic activities. In the medical practice, S.D.O. forms are often handwritten and many fields are generally left empty thus definitely providing only a small amount of all available information.

The process of compiling S.D.O. can be intensively automated by means of the proposed approach. In particular an RFID installed into the patient's wristband, when passing beside a receiver opportunely positioned in the proximity of a gate, can automatically inform the Hospital Information System about patient moving across hospital units. Three use cases have been here reported according to well-known Unified Modelling Language (UML) notation (Figure 6-11). They account for describing at an abstract level the automation of S.D.O. compiling activity. Use case diagrams are completed each one with a sequence diagram depicting the corresponding action flow.

- Patient Identification: this activity starts upon patient entrance in hospital for hospitalization. Medical staff (for example in first aid unit) identifies the patient and transmits patient data to the Hospital Information System. Consequently, patient id assigned by the system is copied onto an RFID tag hosted in a wristband which is then delivered to the patient to track all its hospital stay.

- Patient Internal Transfer: during hospitalization, the patient is bedded in one or more units depending on medical treatments (analysis, medical exams, surgery) he/she receives. Providing the gates of hospital units with an RFID reader, the entry/exit event of an RFID-equipped patient becomes straightforward. Each reading triggers an event that writes in the Hospital database updating the patient localization.

- Patient receives treatment: the medical doctor, after a medical visit or a surgical operation, writes the performed treatment on the patient's RFID by means of specific interfaces (for example a handheld device). This information is temporary stored into the RFID memory until a reading event, run by the passage through an RFID reader enhanced gate, causes data to be flushed towards the S.D.O. In this way, writing tasks are performed locally and remote database updating is managed asynchronously

\section{Conclusion}

In this chapter the improvement resulted by RFID-based modelling for the clinical risk management has been discussed. The comparison between barcode-based healthcare systems and RFID technologies has shown the possibilities for a significant process reengineering which would represent an essential key to efficacy and efficiency increase in personalized healthcare services. In this view, the new model is centred around the idea of patient as an active element in the clinical process, thus overcoming limits imposed by the concept of medical record. In addition to traceability requirements, an RFID tag (positioned, for example, in the patient's wristband) provides a real-time event trigger mechanism, which can be very useful when a complete overview on the instantaneous state of the healthcare processes is needed. A real-world example of how RFID technology may be integrated within the healthcare processes has been also presented and discussed. 
In conclusion, throughout the text several benefits in using RFID technology in the hospital environment have been considered. They can be classified into two main categories: Benefits being tangible, measurable and attributable to a reduction in "cost" and Intangible benefits. In the first group there are the benefits related to the efficiency and referable to the increasing of high quality processes and, consequently, a reduction of resources committed to solving the problems introduced by real or potential non-compliance concerns (corrective and preventive actions). Some classic examples of error, which should be corrected or prevented, refer, for instance, to the surgeon operating on the wrong side because of a transcription error or the doctor and the professional operator administering the incorrect therapy due to a homonymy of patients' names.

The intangible benefits can be subgrouped into further categories:

1. Benefits related to a strengthening of the information system;

2. Subjective benefits such as:

a) Patient satisfaction in feeling more safe;

b) Greater confidence in the structure by the patient;

c) A better sense of belonging of staff due to the awareness that the system manages to avoid a large number of human errors; these factors are evaluated on the basis of the increase in the reference values of the quality indicators perceived by the users / patients and staff belonging to the structure (clinical audit).

3. Compliance with the requirements of Law for safe identification and treatment of data in accordance with the right to privacy of the patient;

4. Benefits attributable to the healthcare facility.

In light of what has been stated so far the authors have been considering the perspective that in the next future RFID-based models focused on a "patient-centric" view will be at the base of intensive healthcare process reengineering. 


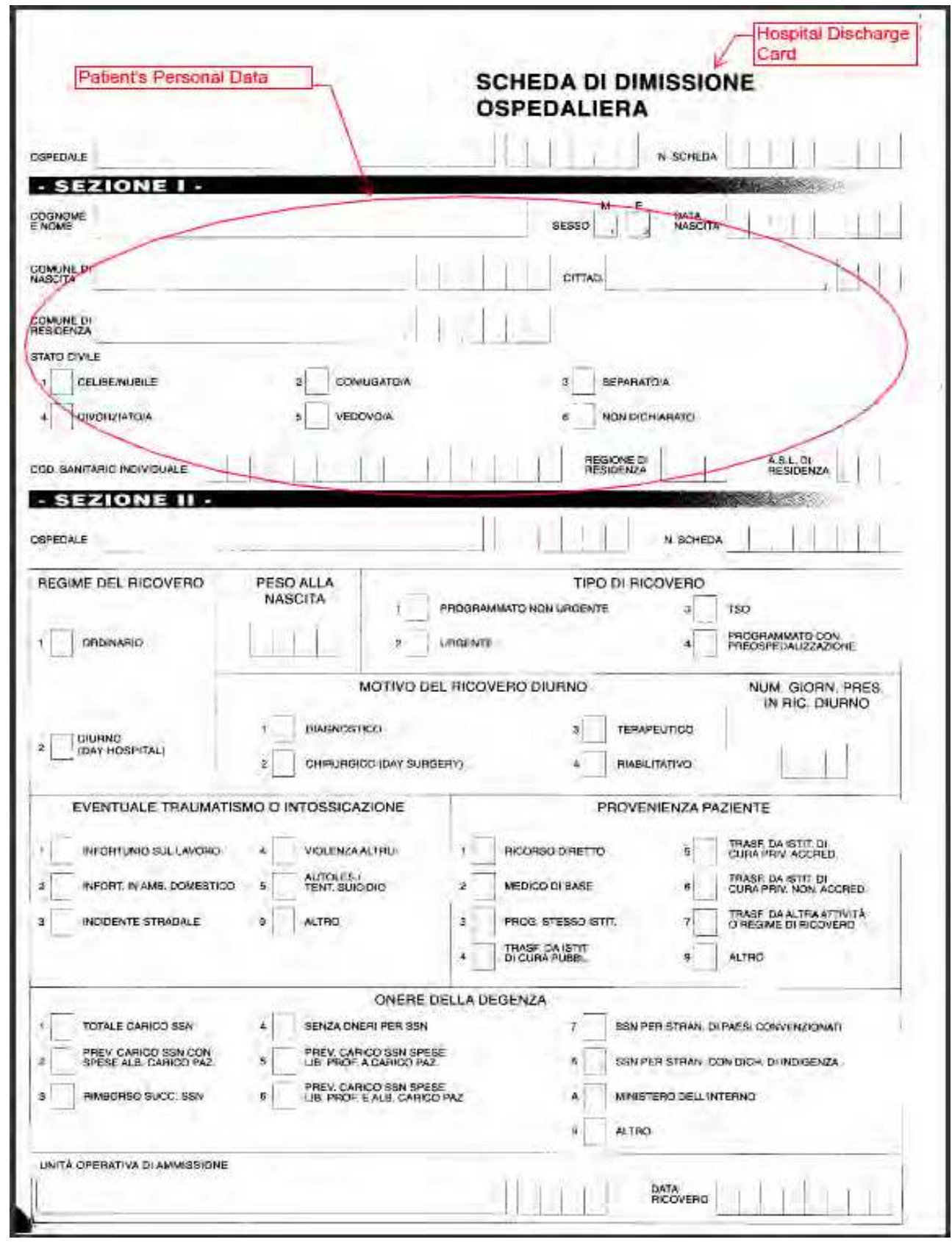

Fig. 4. - The patient's identification page of a S.D.O. document. This is the first page of a SDO and contains almost patient's personal data and summary information about hospitalization. 


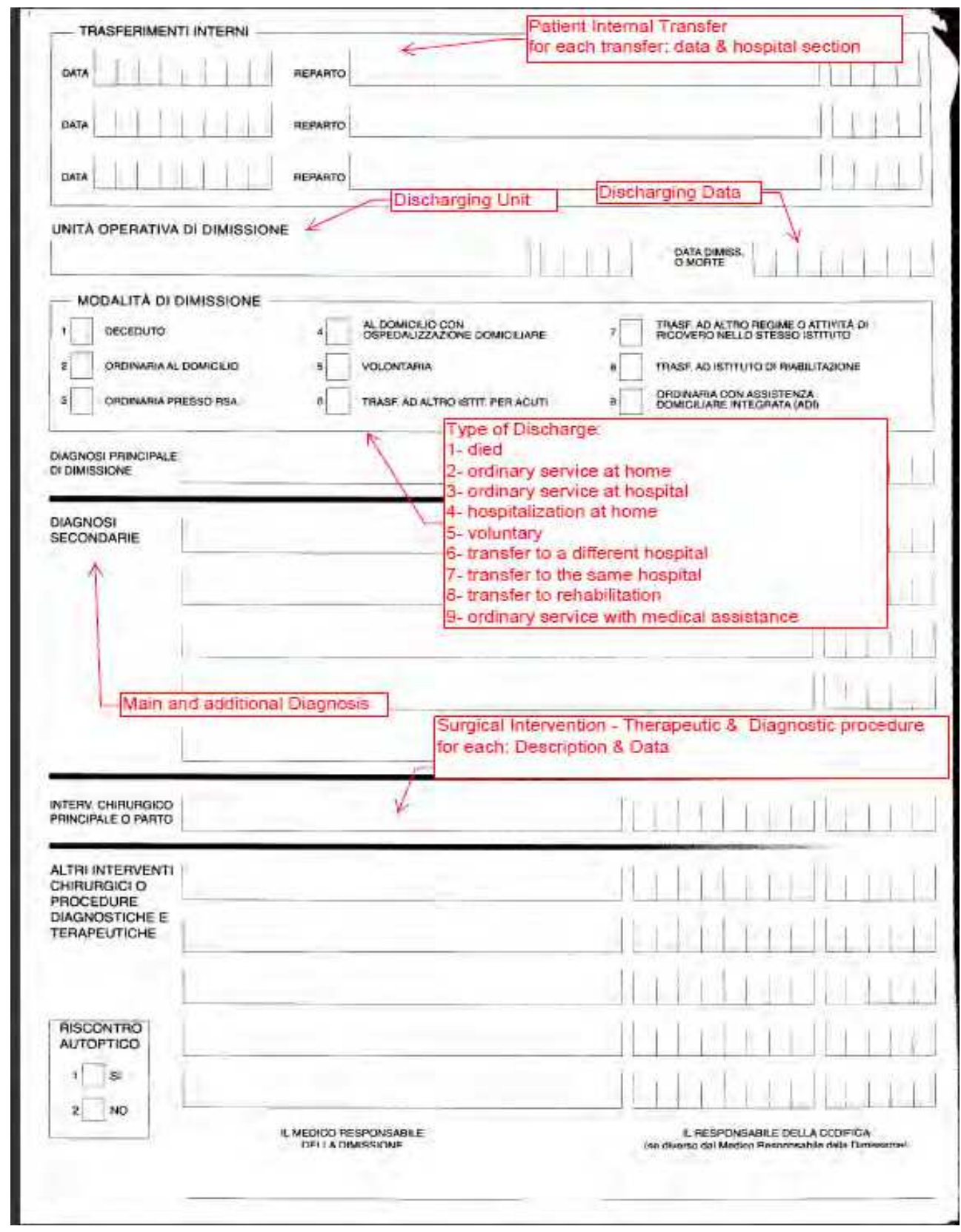

Fig. 5. - The patient's activity page of a S.D.O. This is the second page of a SDO and contains information about all the hospitalization, surgical, therapeutic and diagnostic procedure regarding a patient. 


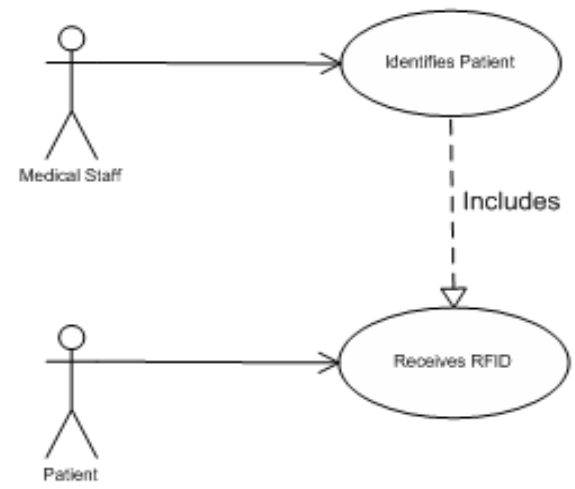

Fig. 6. - UML Use case of patient identification. This use case defines the filling process of the SDO for what concerns patient's identification (S.D.O. first page)

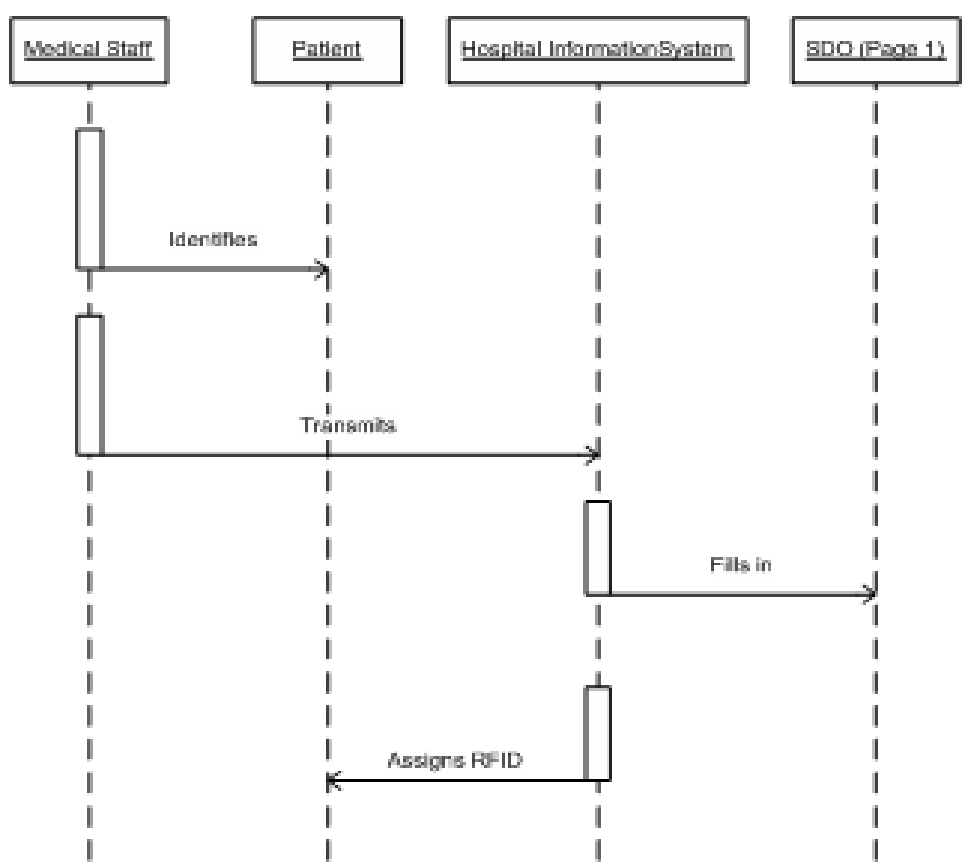

Fig. 7. - UML Sequence Diagram of patient identification. This UML diagram analyzes the sequences of patient identification, data acquisition and RFID assignation. 


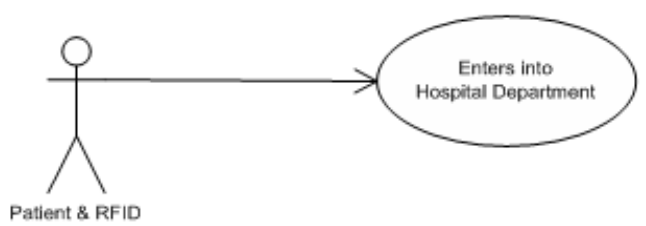

Fig. 8. - Use case of patient activity monitoring. This use case defines the filling process of the S.D.O. second page regarding the hospital departments in which the patient transits.

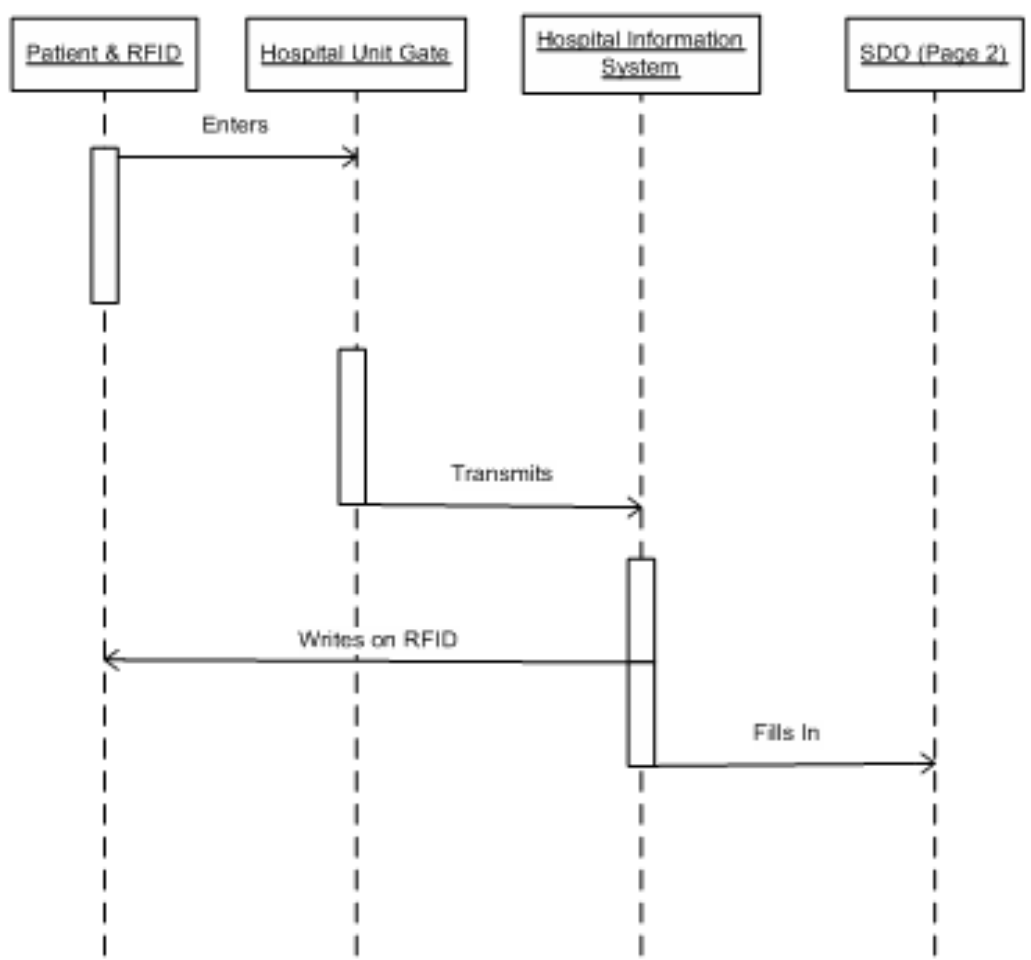

Fig. 9. - Sequence diagram of patient activity monitoring. This sequence diagram analyzes how information about the patient transit in hospital departments is managed by the proposed systems. 


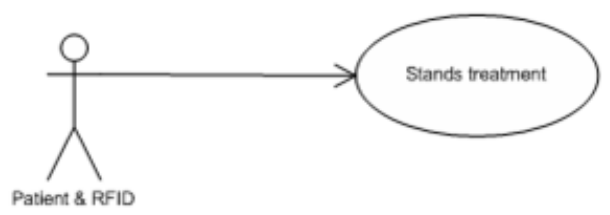

Fig. 10. - Use case of patient activity monitoring. This use case defines the filling process of the S.D.O. second page regarding the treatments (surgical, therapeutics and diagnostics) related to patient

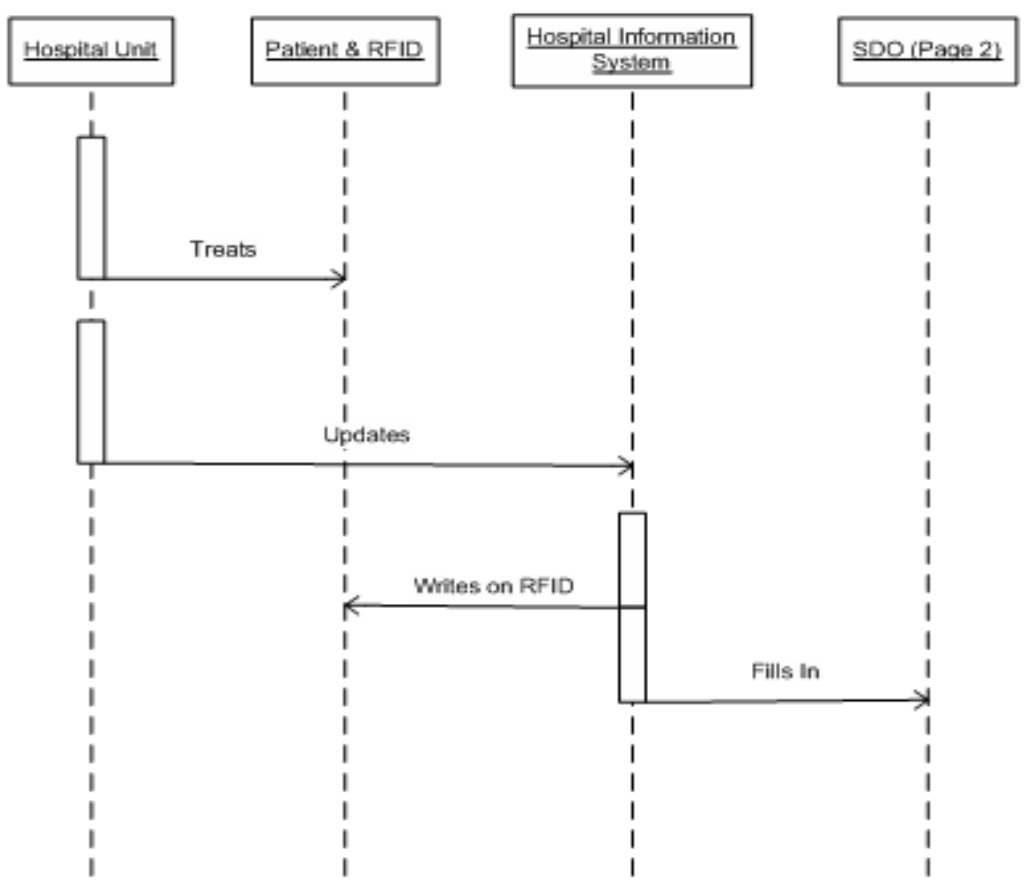

Fig. 11. - Sequence diagram of patient activity monitoring. This sequence diagram analyzes the sequence of operations in correspondence with the treatments of a patient (surgical, therapeutics and diagnostics).

\section{References}

Al Nahas, H. \& Deogun, J.S. (2007). Radio Frequency Identification Applications in Smart Hospitals, Proceedings of the 28th IEEE International Symposium on Computer-Based Medical Systems (CBMS07), pp. 337-342, ISBN 1063-7125, Maribor, Slovenia, June 2007, IEEE 
Anguilar, A.; van der Putten, W. \& Maguire, G. (2006). Positive Patient Identification using RFID and Wireless Networks, Proceedings of the 11th Annual Conference and Scientific Symposium, Health Informatics Society of Ireland, November 2006

Bates, D.; Cohen, M.; Leape, L.; Overhage, M.; Shabot, M. \& Sheridan, T. (2001). Reducing the frequency of errors in medicine using information technology. Journal of the American Medical Informatics Association, Vol. 8, No. 4, July/August 2001, pp. 299308, ISSN 1067-5027

Brenni, S.; Piazza, T. \& Farinella E. (2007). La tracciabilità del paziente in strutture ospedaliere, Notiziario dell'Istituto Superiore Sanità, Vol. 20, No. 9, pp. 3-8, ISSN 03949303

Clarke, D. \& Park, A. MD (2006). Active-RFID System Accuracy and ist Implications for Clinical Applications, Proceedings of the 19th IEEE Symposium on Computer-Based Medical Systems (CBMS06), pp. 21-26, ISBN 0-7695-2517-1, Salt Lake City, UT, USA, June 2006, IEEE

Dario, R.; Quarto, A. \& Di Lecce, V. (2007). Modello per la valutazione del rischio clinico: codice a barre vs RFID, Proceedings of @ITIM 2007 8th National Congress, pp. 31-36, ISBN 978-88-95614-02-1, Bari, Italy, December 2007, @ITIM

Dario, R.; Giove, A. \& Calò, M. (2007). Intelligent Software Agency for Developing Multipurpose Health Care Information Systems, Proceedings of @ITIM 2007 8th National Congress, pp. 157-162, ISBN 978-88-95614-02-1, Bari, Italy, December 2007, @ITIM

Di Lecce, V.; Quarto, A.; Dario, R \& Calabrese, M. (2008). Data Modelling for Complex Reality: an Application to the Clinical Risk Management, The 17th IASTED International Conference on Applied Simulation and Modelling, pp. 196-200, ISBN 978-088986-731-4, Corfu, Greece, June 2008, IASTED

Friesner, D.; Neufelder, D.; Raisor, J. \& Khayum, M. (2005). Benchmarking patient improvement in physical therapy with data envelopment analysis, International Journal of Health Care Quality Assurance, Vol. 18, No. 6, pp. 441-457, ISSN 0952-6862

Fuhrer, P. \& Guinard, D. (2006). Building a Smart Hospital using RFID technology, Proceedings oft he 1st European Conference on eHealth (ECEH06), pp. 131-142, Fribourg, Germany, October 2006, GI-Edition - Lecture Notes in Informatics

Gruber, T.R. (1993). A Translation Approach to Portable Ontology Specifications, Knowledge Acquisition, Vol. 5, No. 2, pp. 199-220, ISSN 1042-8143

Jiang, M.; Fu, P.; Chen, H.; Chen, M.; Xing, B.; Sun, Z.; Deng, P.; Wang, G.; Xu, Y. \& Wang, Y. (2005). A Dynamic Blood Information Management System Based on RFID, Proceedings of the 2005 IEEE Engineering in Medicine and Biology 27th Annual Conference, pp. 546-549, ISBN 0-7803-8741-4, Shanghai, China, September 2005, IEEE

Lehmann, L.S.; Puopolo, A.L.; Shaykevich, S \& Brennan, T.A. (2005). Iatrogenic events resulting in intensive care admission: frequency, cause, and disclosure to patients and institutions, American Journal of Medicine, Vol. 118, No. 4, pp. 409-413, ISSN: 0002-9343

Liao, P.; Liu, L.; Kuo, F. \& Jin, M. (2006) Developing a Patient Safety Based RFID Information System - An Empirical Study in Taiwan, Proceedings of the IEEE International Conference on Management of Innovation and Technology, pp. 585-589, ISBN: 1-4244-0147-X, Singapore, China, June 2006, IEEE 
Ni, L.M.; Liu, Y.; Lau, Y.C. \& Patil, A.P. (2004). LANDMARC: Indoor Location Sensing Using Active RFID, Wireless Networks, Vol. 10, No. 6, November 2004, pp. 701-710, ISSN 1022-0038

Osmon, S.; Harris, C.B.; Dunagan, W.C.; Prentice, D.; Fraser, V.J. \& Kollef, M.H. (2004). Reporting of medical errors: an intensive care unit experience, Critical Care Medicine, Vol. 32, No. 3, pp. 727-733, ISSN 0090-3493

Perilli, G. (2007). Le morti evitabili, ASL/BA - Divisione Territoriale ex AUSL BA editore, Barletta (BA), Italy

Perrin, RA. \& Simpson, N. (2004). RFID and Bar Codes - Critical Importance in Enhancing Safe Patient Care, Journal of Healthcare Information Management, Vol. 18, No. 4, November 2004, pp. 33-39, ISSN 1099-811X

Rapellino, M. (2005). Anatomia dell'errore: errore personale o errore di sistema?, Atti del Simposio dalla Qualità percepita alla percezione dell'errore medico. Metodologia integrata per l'individuazione dell'errore medico, Torino, Italy, Ottobre 2005, http://web.infinito.it/utenti/f/fappto/errore_medico_2005/rapellino_ab.html

Roark, DC \& Miguel, K. (2006). RFID: bar coding's replacement?, Nursing Management, Vol. 37, No. 2, pp. 28-31, ISSN 0744-6314

Rothschild, J.M.; Landrigan, C.P.; Cronin, J.W.; Kaushal, R.; Lockley, S.W.; Burdick, E.; Stone, P.H.; Lilly, C.M.; Katz, J.T.; Czeisler, C.A. \& Bates, D.W. (2005). The Critical Care Safety Study: The incidence and nature of adverse events and serious medical errors in intensive care, Critical Care Medicine, Vol. 33, No. 8, August 2005, pp. 16941700, ISSN 0090-3493

Sangwan, R.S.; Qiu, R.G. \& Jessen, D. (2005). Using RFID Tags for Tracking Patients, Charts and Medical Equipment within an Integrated Health Delivery Network, Proceedings of the 2005 IEEE Symposium on Networking, Sensing and Control, pp. 1070-1074, ISBN 0-7803-8812-7, Malverna, PA, USA, March 2005

Sun, P.R.; Wang, B.H. \& Wu, F. (2008). A New Method to Guard Inpatient Medication Safety by the Implementation of RFID, Journal of Medical Systems, Vol. 32, No. 4, August 2008, pp. 327-332, ISSN 0148-5598

Turner, C.L.; Casbard, A.C. \& Murphy, M.F. (2003). Barcode technology: its role in increasing the safety of blood transfusion, Transfusion, Vol. 43, No. 9, pp. 1200-1209, ISSN 0041-1132

Wicks, A.M.; Visich, J.K. \& Li, S. (2006). Radio frequency identification applications in healthcare, International Journal of Healthcare Technology and Management, Vol. 7, No. 6, pp. 522-540, ISSN 1368-2156

Wu, B.; Liu, Z.; George, R. \& Shujace, K.A. (2005), eWellness: Building a Smart Hospital by Leveraging RFID Networks, Proceedings of the 2005 Engineering in Medicine and Biology 27th Annual Conference, pp. 3826-3829, ISBN 0-7803-8741-4, Shanghai, China, September 2005, IEEE

Young, D (2006). Pittsburgh Hospital combines RFID, bar codes to improve safety, American Journal of Health-System Pharmacy, Vol. 63, No. 24, December 2006, pp. 2431-2435, ISSN 1079-2082 


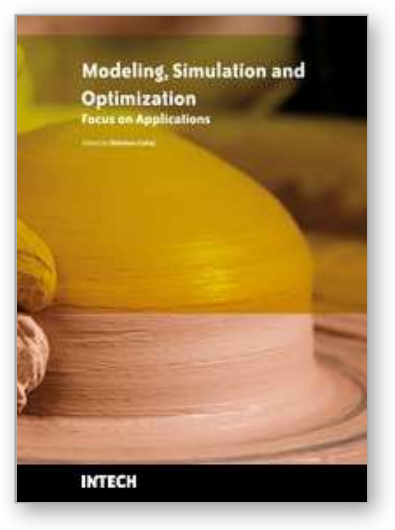

\author{
Modeling Simulation and Optimization - Focus on Applications \\ Edited by Shkelzen Cakaj
}

ISBN 978-953-307-055-1

Hard cover, 312 pages

Publisher InTech

Published online 01, March, 2010

Published in print edition March, 2010

The book presents a collection of chapters dealing with a wide selection of topics concerning different applications of modeling. It includes modeling, simulation and optimization applications in the areas of medical care systems, genetics, business, ethics and linguistics, applying very sophisticated methods. Algorithms, 3-D modeling, virtual reality, multi objective optimization, finite element methods, multi agent model simulation, system dynamics simulation, hierarchical Petri Net model and two level formalism modeling are tools and methods employed in these papers.

\title{
How to reference
}

In order to correctly reference this scholarly work, feel free to copy and paste the following:

Vincenzo Di Lecce, Marco Calabrese, Alessandro Quarto and Rita Dario (2010). Modelling the Clinical Risk: RFID vs Barcode, Modeling Simulation and Optimization - Focus on Applications, Shkelzen Cakaj (Ed.), ISBN: 978-953-307-055-1, InTech, Available from: http://www.intechopen.com/books/modeling-simulation-andoptimization-focus-on-applications/modelling-the-clinical-risk-rfid-vs-barcode

\section{INTECH}

open science | open minds

\section{InTech Europe}

University Campus STeP Ri

Slavka Krautzeka 83/A

51000 Rijeka, Croatia

Phone: +385 (51) 770447

Fax: +385 (51) 686166

www.intechopen.com

\section{InTech China}

Unit 405, Office Block, Hotel Equatorial Shanghai

No.65, Yan An Road (West), Shanghai, 200040, China

中国上海市延安西路65号上海国际贵都大饭店办公楼 405 单元

Phone: +86-21-62489820

Fax: +86-21-62489821 
(C) 2010 The Author(s). Licensee IntechOpen. This chapter is distributed under the terms of the Creative Commons Attribution-NonCommercialShareAlike-3.0 License, which permits use, distribution and reproduction for non-commercial purposes, provided the original is properly cited and derivative works building on this content are distributed under the same license. 